\title{
Cumplimiento del Programa de Gobierno de la Alianza en el sector salud
}

\section{Cecilia Sepúlveda C.}

Facultad de Medicina, Universidad de Chile

csepulve@med.uchile.cl

Este artículo evalúa los primeros quince meses del gobierno de Sebastián Piñera y el grado en que ha cumplido con su programa en el sector de la salud. Para ello, se analizan los avances en cinco áreas prioritarias. El argumento principal es que la estrategia del Ministerio de Salud de anunciar grandes cambios ha sido insatisfactoria, porque dichos anuncios no han sido efectivos en la solución para los problemas del país y porque carecen de respaldo político.

Palabras Clave: salud, políticas públicas, Chile.

\section{The Alianza Government's fulfilment of CAMPAIGN PLEDGES IN THE HEALTH SECTOR}

This article evaluates the first fifteen months of the Sebastian Piñera government and the degree to which it has fulfilled its program in the health sector. Progress in five priority areas is analyzed. The principal argument is that the Ministry of Health's strategy of announcing substantial changes has been unsatisfactory, as the lack of political backing has impeded following up these declarations with concrete policies.

Keywords: health, public policy, Chile. 


\section{La exitosa experiencia del Servicio Nacional de Salud (SNS)}

Creado en 1952, el SNS fue determinante en los avances de la medicina y en la equidad del acceso a la atención, cubriendo todas las regiones del país, incluyendo las rurales y las zonas más alejadas, entregando acciones integrales de salud, como prevención, fomento, medicina curativa y rehabilitación. Creó programas nacionales específicos como los de atención del embarazo y del parto, materno-infantil, de tuberculosis, de enfermedades cardiovasculares y otras, lo que permitió en pocos años una disminución muy significativa de la mortalidad materna, infantil y de enfermedades infecciosas y crónicas no transmisibles. El SNS se constituyó, en el contexto mundial, como un ejemplo del modelo de salud de un Estado benefactor. Mantuvo una fuerte coordinación con la Universidad de Chile, con sedes en las principales regiones del país, desarrollando una fructífera relación docente-asistencial y la formación de recursos humanos en salud de acuerdo a las necesidades del país.

Cabe destacar que en el año 1931, en el país, sólo el 33,5\% de los partos de nacidos vivos ocurrían en hospitales, naciendo el resto de los niños en sus casas, asistidos por personas de la comunidad o por parteras. En 1951 esta cifra se había casi duplicado, llegando al 60,2\%. En el año 1975, la atención de partos hospitalarios alcanzaba el $87,4 \%$ a nivel nacional y quince años más tarde aumentaba a un 97,8\%, alcanzando el año 2000 el 99,7\%, cifra que se mantiene hasta la fecha.

\section{La reforma de la dictadura militar}

Tras el golpe militar de 1973 no hubo cambios en las políticas de salud hasta 1979 cuando, por decreto del gobierno, se reorganizó el SNS y establecieron las bases del actual Sistema Nacional de Servicios de Salud (SNSS). Se crearon 27 servicios de salud descentralizados que pasaron a ser los continuadores legales del SNS y también del Servicio Médico Nacional de Empleados (SERMENA) creado en 1937. El financiamiento de la medicina curativa y de los servicios de salud en su conjunto se radicó en una nueva institución, el Fondo Nacional de Salud (FONASA). De esta manera, se unificó el sistema y, al mismo tiempo, se descentralizó.

Otro decreto, de 1980, traspasó los consultorios primarios de salud a las municipalidades, entregando su propiedad y bienes a las comunas respectivas. En 1981 se crearon las Instituciones de Salud Provisional (ISAPRE), entidades que otorgan 
prestaciones de salud privadas a los cotizantes del sistema, posibilitando una importante expansión de la salud privada. Se estableció una cotización obligatoria para todos los trabajadores, cualquiera fuese su remuneración, equivalente al $7 \%$ de su sueldo imponible. De este modo, quienes podían acceder a la salud privada cotizaban en las ISAPRE y la gran mayoría de más bajos ingresos, en FONASA.

Se estableció así un sistema mixto de salud en el país que se compone de dos subsistemas que actúan separadamente: el público y el privado, operando con grandes desigualdades entre sí y también al interior de cada uno.

A comienzos de la década de los 90, el deterioro de los establecimientos de la red hospitalaria, en particular de los hospitales, era creciente. El déficit de camas era muy importante en el subsistema público. Por otra parte, tanto el gasto público total como el aporte fiscal a éste habían llegado a sus niveles históricos más bajos. Durante el período 1974-1990 se experimentó una caída sistemática del gasto público en Salud como porcentaje del PIB, pasando de alrededor de un 3,3\% el año 1974 a menos de 2,0\% el año 1990.

Esta reducción del PIB en paralelo a la desintegración del sistema público de salud, la centralización de los fondos públicos de la seguridad social en FONASA y la creación de las ISAPRE generó un escenario de completo desfinanciamiento del sistema público a comienzos de los años 90, el que tenía como objetivo la privatización de la salud.

\section{La reforma de la salud en los gobiernos de la Concertación}

Con el retorno de la democracia al país en 1990, el marco jurídico y legal del área de la salud se mantuvo inalterable, dado que para hacer reformas sustanciales se requiere de quórum calificado en el Congreso, es decir, ser aprobadas al menos por $2 / 3$ de éste.

Entre los años 2002 y 2005 los gobiernos de la Concertación plantean una reforma sustantiva al sector salud, con cuatro Leyes que han permitido cambios de gran importancia, los cuales se han ido produciendo paulatinamente, encontrándose esta reforma en pleno desarrollo al momento de asumir el actual gobierno de la Alianza por Chile, el 11 de marzo de 2010. 
Los ejes principales de este proceso de transformación están dados en la Ley de Autoridad Sanitaria y Gestión y la Ley de Régimen General de Garantías en Salud, conocida como Ley AUGE, ambas publicadas en el año 2004. La primera separó las funciones de provisión de servicios de salud, de las de regulación y fiscalización de la normativa sanitaria, creando las Subsecretarías de Salud Pública y la de Redes Asistenciales, así como la Superintendencia de Salud; y redefinió al servicio de salud como el gestor de la red asistencial, dotando de mayor flexibilidad a la gestión de las redes con la creación de establecimientos autogestionados en red.

La Ley AUGE creó un sistema de garantías explícitas de acceso, oportunidad, calidad y protección financiera para un conjunto de problemas de salud prioritarios; que entraron en vigencia a contar de julio del año 2005, para el sector público y privado. Inicialmente se incorporaron 25 problemas de salud al AUGE, aumentándose progresivamente hasta llegar a 66 en la actualidad. Se proyecta incorporar otros catorce problemas de salud prioritarios hasta llegar a un total de 80. La reforma no innovó respecto de los consultorios de atención primaria, los que continúan dependiendo de los municipios. Esto genera inequidad entre una mayoría de comunas pobres y las ricas, ha incrementado el gasto administrativo y la dificultad de mantener la calidad y la efectividad de las acciones sanitarias.

Tras seis años de implementación, la reforma de salud ha puesto de manifiesto la falta de preparación de la red pública para dar satisfacción a la demanda creciente de la población. Si bien se ha otorgado una enorme cantidad de atenciones AUGE, se ha generado una inaceptable discriminación AUGE - No AUGE, y ha quedado en evidencia un manifiesto déficit de camas y de recursos humanos. Esto cobra especial relevancia en la Atención Primaria de Salud (APS), puerta de acceso de los beneficiarios del sistema público a la atención de salud, donde existe un déficit de prácticamente el $50 \%$ de los médicos y de otros profesionales de la salud, a lo que se suma la alta rotación de estos profesionales, producto de los bajos incentivos. Se ha constatado que sólo alrededor del 40\% de los médicos de consultorios municipalizados alcanzan una antigüedad mayor de un año en esos establecimientos y el 60\% no supera los tres años.

Es necesario destacar que durante todos los gobiernos de la Concertación se avanzó de manera sustantiva en el necesario proceso de recuperación de la infraestructura y funcionamiento del sistema público de salud y se mejoró la regulación del sistema privado de aseguramiento y de atención. 
Entre 1990 y 1999 se produjo un incremento significativo del Gasto Público Total, llegando a un 2,9\%. Ello se debió a un aumento del gasto fiscal, lo que permitió que recién el año 2008 el gasto público en salud, como porcentaje del PIB, alcanzara los niveles que el país tenía 34 años atrás, aunque en condiciones bastante diferentes en todos los planos.

Entre FONASA e ISAPRE el gasto actual asciende a un 5,5\% del PIB. Las ISAPRE gastan un $47 \%$ de éste, para atender al 17\% de la población, en tanto que el sistema público gasta el 53\% para atender al 73\%. En términos de gasto per cápita, este gasto es la mitad de lo que gastan las ISAPRE en sus beneficiarios y también es menos de la mitad de lo que otros países de parecido nivel de desarrollo a Chile gastan en sus sistemas públicos.

El Presupuesto del Sector Salud para el año 2010 ascendió a $\$ 3.320 .408$ millones, con un crecimiento del $8,7 \%$ respecto del año anterior. Un $17 \%$ estuvo destinado a reforzar la atención primaria y un 14\% a mantener en ejecución la cartera de inversiones en infraestructura y equipamiento de la red asistencial. El presupuesto en salud ha crecido en un $10 \%$ en promedio en los últimos diez años, duplicándose en el decenio, como se observa en el siguiente gráfico.

Gráfico 1

Presupuesto sector Salud 2001-2010

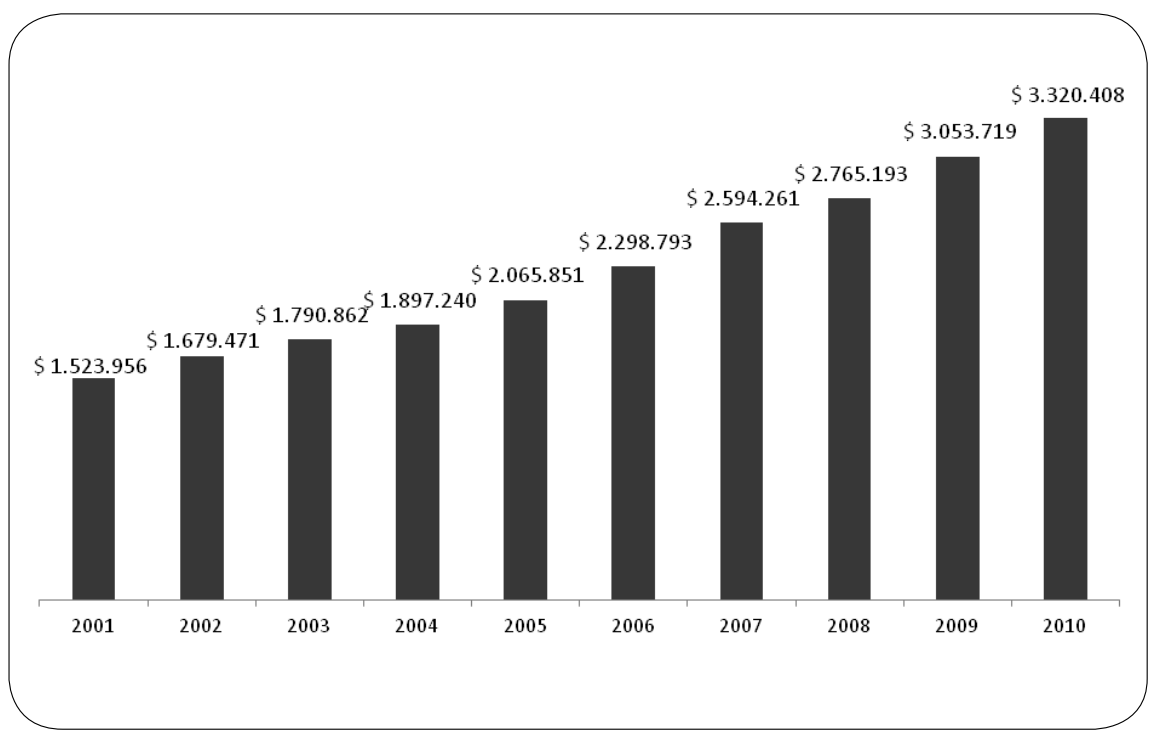

Fuente: MINSAL 2010 


\section{El Gobierno de la Alianza en Salud}

El programa de gobierno de Sebastián Piñera en el sector salud, está estructurado en torno a cinco ejes, los que reafirmó en su mensaje del 21 de mayo de 2010 y, de algún modo, reiteró y amplió en su último mensaje al país.

Cabe hacer notar que, debido a las consecuencias del terremoto del 27 de febrero de 2010, su programa debió reorientarse a fin de enfrentar dicha situación. De los 180 hospitales públicos, 130 se vieron afectados, 25 de ellos con daños severos; y de las 26.500 camas del sector público, se perdieron 4.200, esto sin contar los daños del sector de atención primaria. Los cinco ejes del programa de gobierno de Sebastián Piñera en salud son:

1. Mejorar la infraestructura con la construcción de 10 hospitales y 76 consultorios vía concesiones, destinar US\$ 620 millones para recuperar, modernizar y ampliar más de 50 hospitales y 200 consultorios, priorizando la dotación de médicos especialistas, medicamentos, inversión en equipamiento y en ambulancias.

Después del terremoto el gobierno informó al país que la construcción hospitalaria, vía plan de inversiones y vía concesiones sería: 6 hospitales que se encuentran en etapa de desarrollo del proyecto: Curicó, Penco-Lirquén, Sótero del Río, SalvadorGeriátrico, La Florida y Maipú, más 7 proyectos en etapa de licitación de las obras civiles: Antofagasta, Calama, Gustavo Fricke, Talca (segunda etapa), Traumatológico de Concepción y Puerto Aysén.

En julio de 2010 se anunció la construcción de 3 hospitales modulares (Parral,Talca Interno y Félix Bulnes), y la de los hospitales Del Salvador y Sótero del Río, todos ellos vía concesión.

Las autoridades de salud improvisaron en la búsqueda de soluciones para reponer la infraestructura dañada por el terremoto. Es así como primero se anunció que la recuperación de camas perdidas se haría en forma rápida a través de la adquisición de hospitales modulares y luego esto fue cambiado por una alternativa de construcción "acelerada" de los mismos. Algo similar ocurrió con los plazos, primero se dijo que esto se haría en tres meses, para finalmente anunciar que se haría en el plazo de un año. Hoy, después de 15 meses, los primeros dos hospitales de construcción acelerada, Félix Bulnes y Talca Interno, acaban de ser inaugurados. 
El gobierno, en esta materia, ha actuado con extrema lentitud, lo que ha significado que los usuarios sean los más perjudicados, ya que aumentaron las listas de espera en las zonas del terremoto y la calidad de la atención ha empeorado.

\section{Realizar una gestión moderna, para el año 2014: habrá como mínimo 45 hospitales autogestionados (en la actualidad habría 11, con mala ex- periencia) para que tengan mayor autonomía y mejores remuneraciones asociadas a la calidad de la atención.}

Este ha sido un tema complejo y por ello se ha debido postergar en diferentes oportunidades su puesta en marcha: La última fecha anunciada para poner en marcha esta medida fue el $1^{\circ}$ de abril de 2011 , siendo imposible llevarla a cabo y ampliándose una vez más los plazos. Recordemos que esta medida forma parte del conjunto de propuestas que aprobó la reforma de salud del año 2005 y está contenida en la ley. Hoy el MINSAL, por la vía de la Subsecretaría de Redes, ha intervenido dichos hospitales y es el Ejecutivo el que está monitoreando la gestión y proponiendo metas de cumplimiento de desempeño asociadas al presupuesto, lo que ha significado problemas serios en algunos hospitales.

Habría que señalar que son muchos los actores involucrados y que se oponen a esta medida, como asimismo indicar que algunas de las experiencias ya en marcha no han sido bien evaluadas por la autoridad.

En primer lugar, y dadas las exigencias explícitas en la ley, no es posible implementar esta medida en muchos de los hospitales que deben entrar en régimen de autogestión y se presentan serias dudas. También se cuestiona que una vez incorporado un hospital al proceso de autogestión, éste pueda seguir respondiendo a los requerimientos exigidos por la red pública de salud para su buen funcionamiento, ya que bien podría ocurrir que privilegiase la atención de patologías "más rentables financieramente y que permiten mayores ingresos", por sobre otras patologías que la población usuaria requiere atenderse y que son "menos rentables".

Por otra parte, los requisitos para acreditarse como establecimiento autogestionado en red son tan amplios y variados, que dificilmente los hospitales pueden cumplirlos, sobre todo, terminar con el endeudamiento crónico del sistema.

También, dado que la selección de los directores de establecimientos autogestionados se realiza a través del sistema de Alta Dirección Pública y las diversas, amplias responsabilidades y competencias que se le exige a los candidatos, como las bajas remuneraciones asociadas, son escasos los candidatos calificados. Todo ello 
ha dificultado implementar esta medida y todo hace pensar que este proceso, de llevarse a cabo, no será fácil.

3. Mejorar la calidad de la atención en salud, priorizando la eliminación de las listas de espera AUGE; para ello se recurrirá a la alianza públicaprivados, creando el Bono AUGE.

El gobierno se propuso terminar en 2 años con las listas de espera de enfermedades AUGE y para ello resolvió que la atención a estos pacientes podría hacerse a través del sistema privado, otorgando un Bono Auge, cuando el sistema público no fuera capaz de satisfacer la garantía de oportunidad tal como lo establece la ley.

Todo indica que esta meta sí se estaría cumpliendo. Cifras oficiales entregadas por FONASA a inicios de este año, y ratificadas en el discurso del Presidente el pasado 21 de mayo, muestran que éstas han disminuido significativamente. Se exigió al Ministerio de Salud terminar con todas ellas en el mes de noviembre del presente año, las que de acuerdo a la versión oficial alcanzaban al mes de mayo a poco más de 50.000 personas.

Además, el gobierno anunció que se pondría en marcha un programa de atención a las personas que han debido esperar entre uno y diez años para conseguir una solución quirúrgica a sus problemas de salud no AUGE, que en total alcanzan a 83.000 personas. Para ello se implementará una política de atención en el sistema público de extensión horaria y la atención de algunas de estas patologías, cuando no pueda hacerse en el sistema público, serán atendidas en el sector privado, a través del sistema de Bonos. Todo indica que esta meta es posible de ser cumplida.

4. Mejorar el sistema de atención primaria, aumentando su capacidad resolutiva, incrementando el per cápita, aumentando los horarios de atención, incorporando médicos especialistas, becas de capacitación para los funcionarios y aumentando los incentivos para retener a los mejores profesionales.

Sin duda este es un problema estructural. Desde que se implementó la municipalización de la atención primaria, ésta presenta falencias muy difíciles de resolver. La falta de médicos y otros profesionales de la salud, la alta rotación de médicos por falta de incentivos, la calidad de la atención, el bajo monto del per cápita asignado, etc., son problemas que deben ser resueltos urgentemente, si es que se quiere fortalecer el sistema de atención primaria.

Por ello uno de los anuncios recientes hechos por el Presidente de la República, en orden a impulsar el AUGE preventivo y la creación de consultorios de excelencia, 
no será posible de cumplirlo si no se señala cómo se va implementar, cuándo y con qué recursos, ya que sin resolver eso en primer lugar, es imposible cumplir con estos anuncios. Lo mismo ocurre con el anuncio de la creación de los consultorios de excelencia, ya que se requiere saber cuántos médicos y profesionales de la salud nuevos se contratarán y cómo serán financiados, pero sobre todo, cómo los motivarán para que trabajen en la atención primaria.

Cabe destacar que la prevención en salud se hace en la atención primaria y hoy no se está haciendo. Para cumplir con las metas de disminuir la obesidad, el consumo de alcohol y drogas, el consumo de sal, promover prácticas de vida saludable, es decir un AUGE preventivo, sólo se puede hacer en este nivel de la atención en salud.

5. Firma de convenios de desempeño con cada uno de los hospitales, fijando metas visibles con el fin de mejorar la "buena atención".

Esta es una meta que está lejos de poder cumplirse durante el actual período presidencial; los esfuerzos realizados hasta ahora han sido menores y no se cuenta hasta ahora con una planificación adecuada y con una clara definición de objetivos.

\section{Otros anuncios del Gobierno ¿en qué están?}

Durante los primeros quince meses de gobierno, la autoridad de salud ha hecho una serie de anuncios y ha enviado diversos proyectos al Parlamento para su tramitación, los que se encuentran en distintas fases de desarrollo. Los más importantes son:

1. Proyecto de Postnatal de seis meses: Cumplido.

2. Proyecto de eliminación del 7\% para los jubilados: Cumplido.

3. Ley de etiquetado de alimentos (llamada también Ley del Súper Ocho): dice relación con la composición nutricional de los alimentos. En principio se había logrado un acuerdo en el Senado (con el senador Girardi), a condición que una vez aprobado, el Presidente de la República aplicara un veto presidencial a dos cuestiones: la prohibición de vender alimentos con alto porcentaje de sal, grasa de mala calidad y azúcar en las universidades y eliminar la publicidad de los sucedáneos de la leche materna. Sin embargo una vez aplicado dicho veto, la oposición no estuvo de acuerdo, ya que vulneraba los acuerdos alcanzados. Según senadores de la oposición, éstos señalaron que se puede llegar a acuerdos para aprobar este proyecto y, entre los aspectos del veto que se está conversando, 
está el que dice relación a las facultades rectoras que se entregan al Ministerio de Salud, "como poder establecer porcentajes máximos de un determinado componente en un determinado alimento y que las empresas puedan adicionar un nutriente que, de alguna manera, pueda inducir a un equívoco por parte del consumidor".

4. Ley sobre fraude de licencias médicas: el día 7 de junio de 2011, fue rechazado por la sala de la Cámara de Diputados, quienes acordaron hacer una consulta al Tribunal Constitucional en relación al artículo $5^{\circ}$ de dicho proyecto, que señala que los médicos pueden ser sancionados por una autoridad administrativa, como es la Superintendencia de Seguridad Social, y no por los Tribunales de Justicia, en caso que los médicos cometan fraude con las licencias médicas. Esta solicitud fue planteada por el Colegio Médico tanto en la Comisión de salud de la Cámara como del Senado.

5. Ley corta de Isapres: actualmente está en punto muerto a la espera de que oposición y gobierno acuerden un proyecto diferente que satisfaga tanto a los usuarios del sistema como a las propias Isapres, quienes manifestaron su desacuerdo con el contenido de dicho proyecto. Cabe destacar que este es un tema pendiente desde que el Tribunal Constitucional se pronunció sobre las actuales tablas de factores con costos elevados en las prestaciones para mujeres, niños menores de dos años y adultos mayores.

En general podemos señalar que el Ministerio de Salud no ha estado a la altura de los requerimientos del país. Su política, al igual que la del gobierno en general, ha sido hacer muchos anuncios, los que no dan cuenta necesariamente de una solución a los problemas más de fondo que el país necesita, como asimismo, hacer anuncios que dificilmente se concretarán por falta de respaldo político.

Lo anterior se refleja de buena forma en la editorial del diario El Mercurio del día 5 de junio de 2011 en que señala: "Las importantes tareas pendientes en el sector salud son tan diferentes de las propuestas que periódicamente formula la máxima autoridad sanitaria, que es aconsejable evitar estas continuas distracciones hacia iniciativas menores", lo cual parece un buen resumen de lo que ocurre actualmente en el sector salud. 